\title{
Targeting Multiple Myeloma Cancer Stem Cells with Natural Products - Lessons from Other Hematological Malignancies
}

Authors

Mark E. Issa, Sylvian Cretton, Muriel Cuendet

Affiliation

School of Pharmaceutical Sciences, University of Geneva, University of Lausanne, Geneva, Switzerland

Key words

cancer stem cells, differentiation, migration, multiple myeloma, resistance, self-renewal

received February 21, 2017

revised April 5, 2017

accepted April 17, 2017

Bibliography

DOI https://doi.org/10.1055/s-0043-109558

Published online May 4, 2017 | Planta Med 2017; 83: 752

760 @ Georg Thieme Verlag KG Stuttgart · New York I

ISSN 0032-0943

Correspondence

Prof. Muriel Cuendet

School of Pharmaceutical Sciences, University of Geneva

Rue Michel Servet 1, 1211 Geneva 4, Switzerland

Phone: + 412237933 86, Fax: + 41223793399

Muriel.Cuendet@unige.ch

\section{ABSTRACT}

Multiple myeloma is characterized by the accumulation of malignant plasma cells in the bone marrow. Multiple myeloma is the second most frequently diagnosed hematological malignancy, predominantly affecting the elderly. Despite recent advances in the development of novel therapies, multiple myeloma remains an incurable malignancy where the majority of patients relapse, develop resistance, and eventually die from the disease. This has been attributed to the fact that conventional therapy currently in use targets mainly the bulk of tumor cells, but not the tumor-initiating cancer stem cells. Cancer stem cells are a highly resistant subpopulation of cells believed to be responsible for the initiation, progression, metastasis, and relapse of cancer. Enormous efforts have been invested in the characterization of cancer stem cells. These efforts led to the characterization of key cellular signaling pathways responsible for conferring stem cell characteristics including self-renewal, differentiation, migratory, survival, and intracellular detoxification capabilities. Targeting these protective mechanisms offers a valuable strategy that may help combat a major driving force behind cancers. The use of natural products offers a promising therapeutic approach for targeting cancer stem cells. In this review, recent advances achieved in the characterization of cancer stem cells derived from hematological malignancies, with a particular focus on multiple myeloma, are discussed and major natural products that target cancer stem cells are presented. As natural products remain an essential source of novel chemical structures and medicinal leads, the exploitation of this immense reservoir is used to draw lessons in targeting multiple myelomacancer stem cells.

\section{Multiple Myeloma - A Brief Introduction}

Up to date, MM accounts for about 20000 new cases and 10000 deaths per year in the USA alone [1]. Numerous therapeutics have been developed for MM treatment including alkalyting agents such as melphalan, corticosteroids such as dexamethasone and prednisone, proteasome inhibitors such as bortezomib and carfilzomib, immunomodulatory agents such as thalidomide, lenalidomide, and pomalidomide, and, more recently, the epigenetic modulator panobinostat [2,3]. The current understanding of MM pathogenesis remains poor in comparison to the outstanding questions frequently raised about MM pathobiology and its almost non-existing curability.
The CSCs paradigm has emerged as a satisfactory explanation behind the low curability of MM. CSCs represent a rare cancer-initiating cell population characterized by self-renewal, differentiation, migration, and resistance $[4,5]$. Thus, heterogeneous tumors that contain both CSCs and bulk tumor cells may benefit from drug regimens that target each population. However, compounds that target MM-CSCs remain largely unidentified, and the identification of such compounds is urgently needed as it may significantly improve the prognosis of MM patients. 


$\begin{array}{ll}\text { ABBREVIATIONS } \\ \text { ABC } & \text { ATP binding cassette } \\ \text { ALDH } & \text { aldehyde dehydrogenase } \\ \text { AML } & \text { acute myeloid leukemia } \\ \text { APL } & \text { acute promyelocytic leukemia } \\ \text { ATRA } & \text { all-trans retinoic acid } \\ \text { BM } & \text { bone marrow } \\ \text { CLL } & \text { chronic lymphoid leukemia } \\ \text { CML } & \text { chronic myeloid leukemia } \\ \text { CSCs } & \text { cancer stem cells } \\ \text { GSCs } & \text { glioblastoma stem cells } \\ \text { H33342 } & \text { Hoechst 33342 } \\ \text { HH } & \text { hedgehog } \\ \text { LSCs } & \text { leukemia stem cells } \\ \text { MM } & \text { multiple myeloma } \\ \text { NSCs } & \text { normal stem cells } \\ \text { PCs } & \text { plasma cells } \\ \text { PTCH } & \text { patched } \\ \text { P-gP } & \text { P-glycoprotein } \\ \text { SCID } & \text { severe combined immunodeficiency } \\ \text { SMO } & \text { smoothened } \\ \text { SP } & \text { side population } \\ \text { TLM } & \text { telomestatin } \\ \end{array}$

\section{Multiple Myeloma Cancer Stem Cells -} A Brief Introduction

The existence of MM-CSCs has been suspected for few decades. A large proportion of MM-derived malignant PCs display a terminally differentiated and quiescent phenotype [6]. This suggests that the remaining distinct minor subpopulation, which is not well differentiated and non-quiescent, may confer tumorigenic characteristics and may be responsible for the fatal relapses observed in MM patients [7, 8].

The earliest known evidence suggesting that MM cells may be functionally heterogeneous was provided by Bergsagel and Valeriote (1968) [9]. In their study, Bergsagel and Valeriote transplanted increasing concentrations of mouse PCs into healthy mouse recipients and found that the number of colonies per recipient spleen increased in a linear manner [9]. Moreover, splenic tumor colonies could be serially engrafted into secondary recipients, thus demonstrating clonogenic and self-renewing properties of the inoculated PCs [9]. Subsequently, an in vitro system using soft agar was developed to grow human suspected tumor CSCs colonies [10]. It was found that MM patients' BM aspirates were capable of colony formation in a manner that was proportional to the number of cells plated. Morphological and functional properties showed that the colonies consisted of immature plasmablasts as well as mature PCs, indicating a differentiation potential of the transplanted cells [10].

In the 1990s, a population of CD19+ B cells termed "clonotypic B cells" was identified in the peripheral blood of MM patients [11].
Clonotypic B cells were capable of functionally differentiating into antibody-secreting PCs in vitro [12]. Clonotypic B cells also showed tumorigenic potential, as demonstrated by engrafting into nonobese diabetic SCID mice, and caused osteolytic lesions [13]. Interestingly, P-gp was highly expressed in the great majority of clonotypic B cells and BM cells from patients previously treated with chemotherapy, but was absent or low in BM cells of untreated myeloma patients [12]. These results had profound clinical implications as clonotypic B cells demonstrated a drug-resistant phenotype and prevailed even after exposure to cytotoxic therapy.

Matsui and colleagues [14] discovered in 2004 that the expression of the cell surface antigen syndecan-1 (CD138), a characteristic of terminally differentiated normal and malignant PCs, but not plasmablasts or earlier B cells, was an important clonogenic marker in both MM cell lines and BM-derived MM samples. Human MM cell lines RPMI 8226 and NCl-H929 contained small (2 to 5\%) subpopulations that did not express CD138. In comparison to CD138 ${ }^{+}$PCs, CD138- cells from both MM cell lines demonstrated a greater clonogenic and self-renewal potential in vitro, as measured by their ability to form colonies in methylcellulose and undergoing serial replating, respectively [14].

Overall, the existence of CSCs has been demonstrated in virtually all cancer types. As such, MM-CSCs have been described as a major driving force behind MM initiation, progression, relapse, and resistance. Thus, MM-CSCs represent a valuable target in the fight against MM, and the identification of compounds capable of eradicating MM-CSCs is urgently needed. One rich reservoir that could be exploited is the natural products reservoir.

\section{Targeting Multiple Myeloma Cancer \\ Stem Cells - Resistance}

From a clinical point of view, the most relevant characteristic of CSCs is resistance. MM-CSCs demonstrated resistance to virtually all available anti-myeloma drugs. Enormous efforts have been directed to target the molecular, genetic, and epigenetic machineries in order to control the resistance aspect in MM-CSCs.

\section{Drug efflux - ATP binding cassette transporters}

Although CSCs can be identified using the expression of cell surface proteins such as CD44, CD166, or CD138, CSCs can also be identified by their ability to efflux fluorescent dyes such as H33342. Cells negative for H33342 appear on the side of the flow cytometry plot and are termed "side population" (SP). An SP phenotype exists because CSCs highly express members of the $A B C$ transporter superfamily, which efflux H33342 and render CSCs negative for this stain. ABC transporters expressed in CSCs play important roles in cellular defense mechanisms by excluding toxic chemicals to the extracellular environment ( $\triangleright$ Fig. 1). Since chemotherapeutics could be substrates for $A B C$ transporters, high $A B C$ transporter expression renders CSCs resistant to therapy [15]. The use of $A B C$ efflux transporter inhibitors, such as verapamil, turns SP myeloma cells positive for H33342 staining. SP myeloma cells displayed important tumorigenic potential, demonstrated elevated levels of ABCG2, and were capable of regenerat- 
ing the entire cell population [16]. Using a similar concept to H33342 SP, MM cell lines NCI-H929 and KMS- 5 were sorted based on their ability to efflux the pluripotent stem cell dye, named CDy1, and two distinct subpopulations, CDy $1^{\text {high }}$ and $C D y 1^{\text {low }}$, were identified. The most differentially expressed gene in the $C D y 1^{\text {low }}$ subpopulation in both MM cells lines was $A B C B 1$, and its expression was negatively correlated with $C D y 1$ staining intensity. $A B C B 1$-overexpressing cells appeared resistant to the proteasome inhibitor CFZ. In addition, CFZ-induced resistance in sensitive MM cells was associated with both increased expression of ABCB1 and increased efflux of CDy1 [17].

Despite the fact that targeting the efflux machinery in MMCSCs seemed an effective strategy for the eradication of CSCs, this area remains under-explored. Perhaps this is because the use of inhibitors of $A B C$ efflux transporters in parallel with chemotherapy failed in the clinic due to adverse effects. However, novel inhibitors are being developed, particularly from natural sources, and these molecules appear to circumvent the toxicities associated with conventional efflux transporter inhibitors.

The effects of Scutellaria baicalensis Georgi (Lamiaceae), a plant used in popular Chinese medicine against numerous human diseases, named Houang Qin, were investigated on the proportion of SP cells within RPMI 8226 cells. The crude ethanolic root extract did not show significant antiproliferative effects, apoptosis induction, or cell cycle arrest in malignant PCs. However, baicalein, wogonin, and, to a lesser extent, baicalin, the major polyhydroxyflavonoid constituents of the ethanolic root extract of S. baicalensis, reduced the H33342-negative SP cells in RPMI 8226 cells, and decreased the expression of ABCG2 in SP cells in vitro ( $\triangleright$ Figs. 2 and 3 ) $[18,19]$. Homology modeling and molecular docking studies indicated that baicalein exhibited similar binding sites and binding modes to the ABCG2 inhibitor fumitremorgin C, a tremorgenic mycotoxin isolated from Aspergillus fumigatus [19].

Similar to MM, the expression of $A B C$ efflux transporters on LSC surfaces renders these cells highly resistant to a wide variety of chemotherapeutic drugs. In one study, KG-1a promyeloblastic leukemia cells were induced to overexpress $A B C$ efflux transporters such as $A B C B 1, A B C G 2$, and $A B C C 8$. The resistant phenotype was reversed by the use of cyclosporin $A$, a known $A B C B 1$ and ABCG2 transporter inhibitor [20]. Salinomycin, a monocarboxylic polyether ionophore antibiotic derived from Streptomyces albus, induced apoptosis in both sensitive and resistant KG-1a cells, indicating that salinomycin warrants an investigation in the context of LSCs that overexpress ABC efflux transporters [20].

\section{Drug metabolism - aldehyde dehydrogenase}

ALDHs are a superfamily of enzymes involved in the detoxification of the intracellular environment. ALDHs are $\operatorname{NAD}(P)^{+}$-dependent enzymes that oxidize both endogenous and exogenous aldehydes to their corresponding weak carboxylic acids [21]. The human ALDH superfamily consists of 19 isozymes that belong to $11 \mathrm{fam}$ ilies and 4 subfamilies. Although ALDH isoforms are widely distributed in the body with relatively more abundance in the kidney and liver, evidence indicates that ALDHs are involved in the protection of both NSCs and CSCs ( $\bullet$ Fig. 1) [21]. ALDH1 has been used as a marker to identify CSCs in virtually all cancers. In addition, increased ALDH1 activity has been associated with a poor prognosis

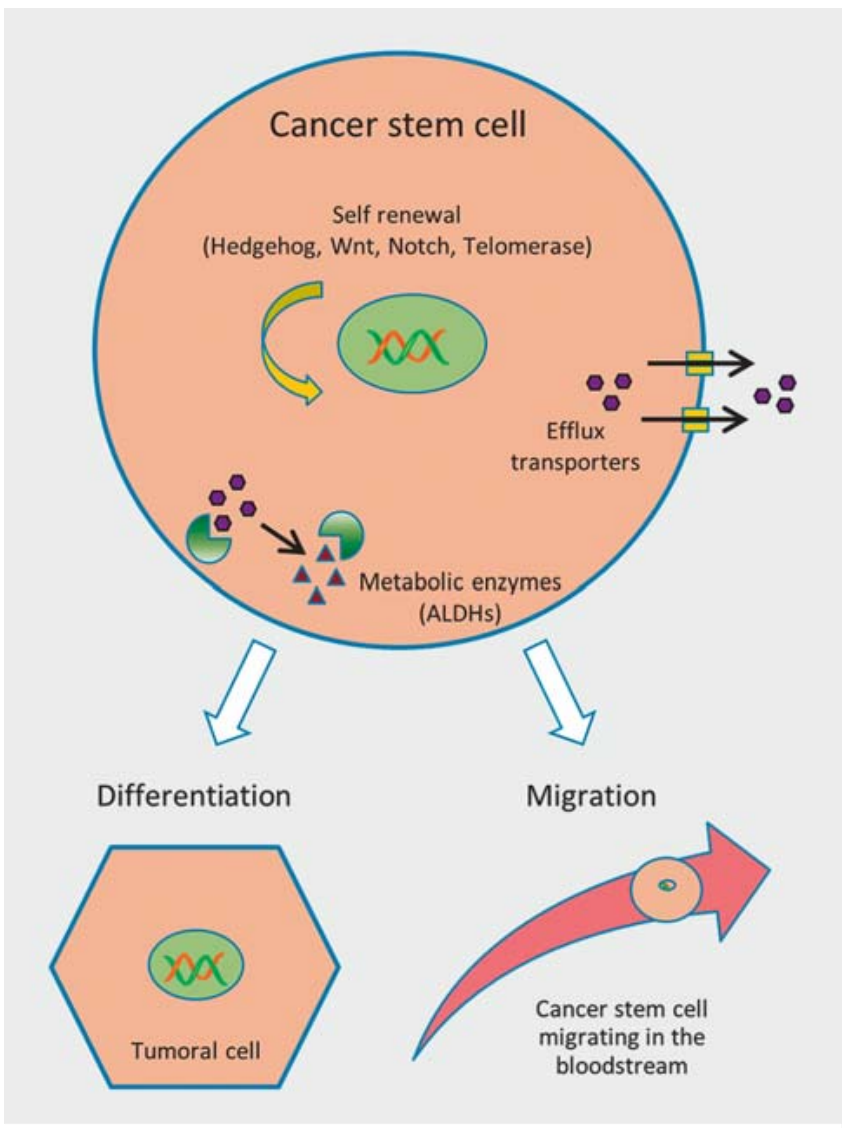

- Fig. 1 General characteristics of CSCs.

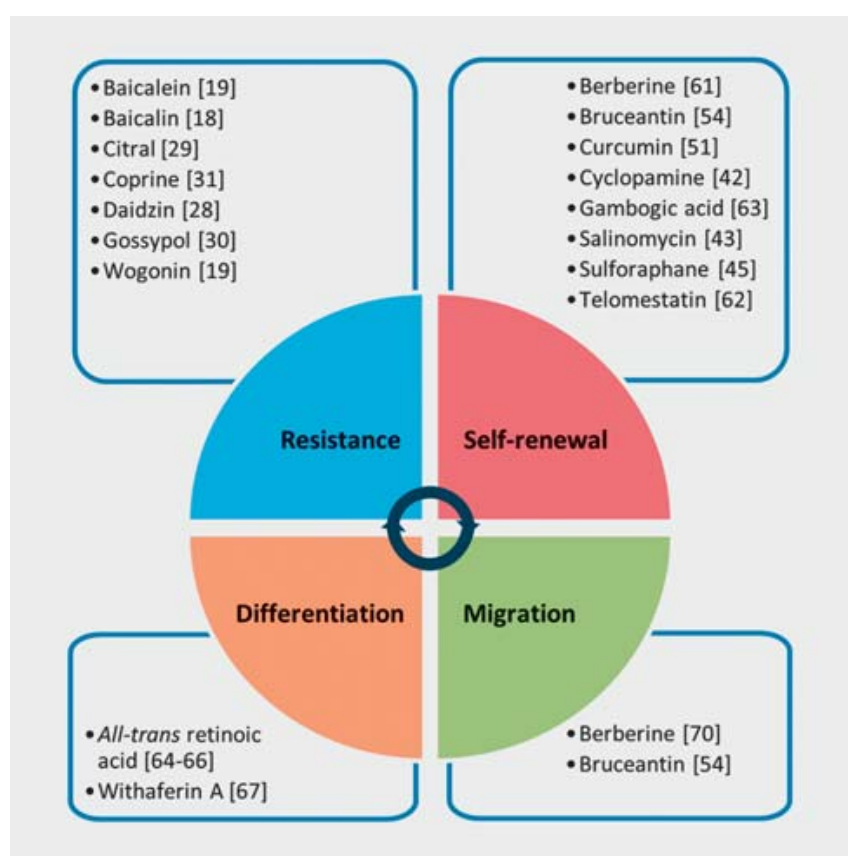

- Fig. 2 Natural products targeting specific CSC characteristics. 

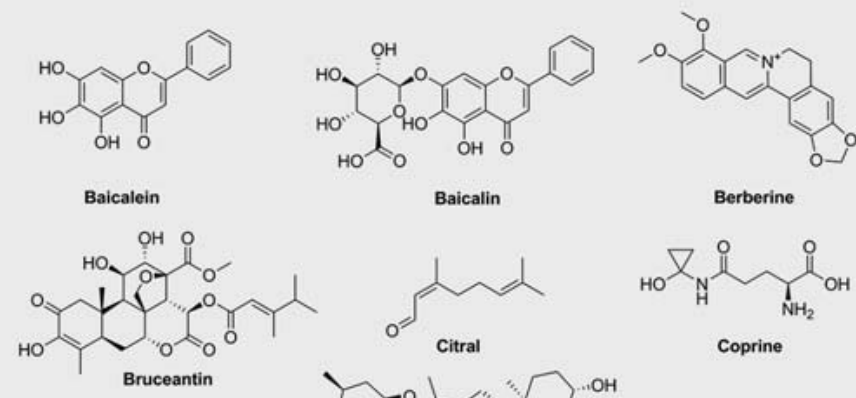

Baicalin

Berberine
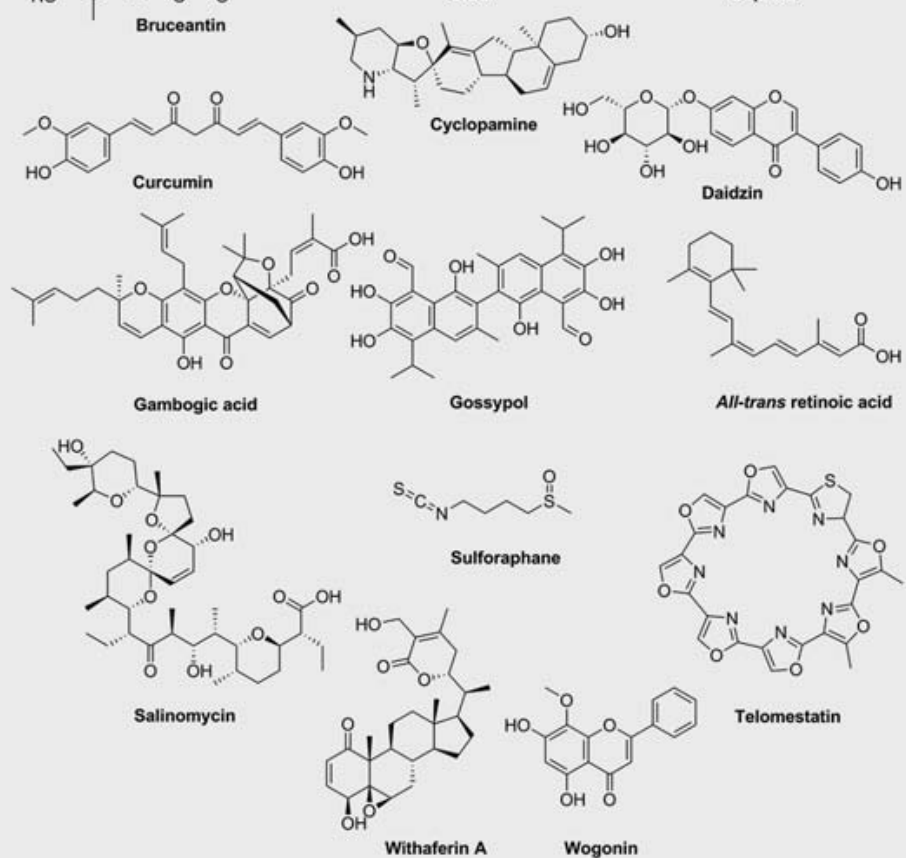

- Fig. 3 Chemical structures of compounds targeting hematological CSCs.

in breast, myeloma, bladder, and prostate cancer patients [21]. In invasive breast cancer ductal carcinomas, 3-10\% of the cells with high ALDH activity (ALDH ${ }^{\text {high }}$ ) were highly tumorigenic compared to ALDH cells with low ALDH activity (ALDH ${ }^{\text {low }}$ ) [22]. This cell population significantly increased following chemotherapy and was sensitized to chemotherapy upon pretreatment with the specific ALDH inhibitor diethylaminobenzaldehyde [23, 24].

Similarly, CD138- MM cells, derived from RPMI 8226 and NCl$\mathrm{H} 929$ cell lines, displayed increased ALDH activity. $\mathrm{CD}^{2} 38^{-} \mathrm{ALDH}^{+}$ cells were substantially more clonogenic than $\mathrm{CD} 138^{+} \mathrm{ALDH}^{-}$cells. CD138- cells gave rise to CD138 ${ }^{+}$cells, and resulted in an upregulation of genes reminiscent of stemness of normal hematopoietic stem cells and LSCs, an indicator which was associated with poor survival in various clinical data sets [25]. Although the important roles that ALDH play protecting CSCs have been clearly characterized, our search indicated that ALDH inhibitors have not yet been tried in MM-CSCs.

Numerous ALDH inhibitors have been developed or discovered from natural sources ( $\triangleright$ Figs. 2 and 3 ) [26, 27]. For instance, daidzin (7-O-glucosyl-4-hydroxyisoflavone), isolated from Pueraria lobata and Glycine max (Fabaceae), is a potent inhibitor of ALDH2 with an IC 50 of $80 \mathrm{nM}$ [28]. Citral (3,7-dimethyl-2,6-octadienal), a mixture of cis and trans isomers named neral and geranial, respectively, are monoterpenes responsible for a strong lemon odor that occurs in some aromatic plants and citrus fruits. Both isomers specifically inhibited the isozymes ALDH1 and ALDH2 [29]. Gossypol, another terpenoid aldehyde extracted from cottonseed (Gossypium spp., Malvaceae), acted as a noncompetitive inhibitor and showed more selectivity towards ALDH3 than ALDH1 and ALDH2 [30]. Coprine or N-(1-hydroxycyclopropyl)-L-glutamine, produced by a fungal species of the genus Coprinus and Coprinopsis (Agaricaceae), was not found to directly inhibit ALDH2, but following in vivo metabolism, its active metabolite 1-amino cyclopropanol irreversibly inhibited ALDH2 [31]. This property explains the "Coprinus syndrome", which is an intoxication due to concomitant ingestion of coprinoid mushrooms with alcohol. Recently, the mode of action of two alkynols with cytotoxic activity, falcarinol and stipudiol, which are encountered in vegetables like carrots (Daucus carota L., Apiaceae) and parsley [Petroselinum crispum (Mill.) Fuss, Apiaceae], was elucidated. The active metabolites of coprine, falcarinol, and stipudiol bind irreversibly via covalent alkylation to the nucleophilic cysteine in the active site of ALDH2 [32]. Another mode of ALDH inhibition was discovered for $\beta$-escin, also named aescin, a triterpene saponin isolated from 
Aesculus hippocastanum L. (Sapindaceae), which does not directly inhibit ALDH1 activity, but rather inhibits its expression in lung tumor cells in a mouse model [33]. With the exception of coprine, which exhibits mutagenic and gonadotoxic effects, thus preventing further development, it would be valuable to test these natural compounds in MM-CSCs and other CSCs [34,35].

\section{Targeting Multiple Myeloma Cancer Stem Cells - Self Renewal}

NSCs and CSCs are characterized by self-renewal, differentiation, and resistance. NSCs depend on self-renewal for tissue homeostasis and regeneration, a property that, in CSCs, maintains the malignancy ( $\bullet$ Fig. 1) $[36,37]$. Several studies have demonstrated that the same signaling pathways that control self-renewal and differentiation in NSCs also do so in CSCs. Therefore, the inhibition of self-renewal has been proposed as a strategy to deplete the malignancy from its CSC reservoir. The signaling pathways that govern self-renewal have been extensively investigated and identified, including, but not limited to, $\mathrm{HH}$, Wnt, and Notch [36, 37]. These pathways are implicated in embryonic development and organ formation. These pathways not only control self-renewal, but also cell fate, migration, and differentiation. Although these pathways appear silenced in most adult tissue, they are reactivated in stem cells following injury or a need to replenish a specific tissue. However, it is important to note that these pathways are only transiently activated following injury, and NSCs revert back to quiescence after the mission of replenishing has been accomplished. A preponderance of human cancers exhibit an aberrant activity in at least one of these pathways [36,37], which are thought to contribute to uncontrolled self-renewal of CSCs as well as promoting a resistant phenotype [8].

\section{Hedgehog inhibition}

The first pathway to be implicated in the maintenance of MMCSCs was the $\mathrm{HH}$ pathway [36]. HH pathway activation starts with the binding of the three ligands, Sonic Hedgehog, Indian Hedgehog, or Desert Hedgehog, to the cell surface receptor PTCH [36]. PTCH is a 12-transmembrane cell surface receptor that upon activation liberates the 7-transmembrane receptor SMO [36]. GLI1, 2, and 3 are released from the SMO-GLI complex, and translocate to the nucleus to modulate the expression of HH target genes [36]. $\mathrm{GLI}$ protein targets include cell cycle regulators such as cyclin D and cyclin E, and tissue development regulators such as PAX [36, 38-40].

Aberrant $\mathrm{HH}$ signaling has been described in a multitude of human cancers and in CSCs. Peacock and colleagues [41] demonstrated that $\mathrm{HH}$ pathway activity is concentrated within the MM stem cell compartment. $\mathrm{HH}$ ligand was found to promote MMCSCs expansion with no effect on differentiation. The blockade of the $\mathrm{HH}$ pathway with cyclopamine, a streroidal alkaloid biosynthesized by Veratrum californicum Durand (Liliaceae), inhibited MM-CSC expansion and induced terminal differentiation ( $\vee$ Figs. 2 and 3) [41]. Interestingly, the effects of the HH pathway on malignant PC growth were negligible, suggesting that $\mathrm{HH}$ pathway activity varies between MM-CSCs and their differenti- ated derivatives [41]. The use of cyclopamine caused significant apoptosis in the primary CD34 ${ }^{+}$(a stem cell surface marker) AML cells after $48 \mathrm{~h}$ [42]. This apoptotic effect was not observed in more differentiated cancer cells such as U937 or HL60, but interestingly, cyclopamine reversed resistance to the anticancer agent cytarabine [42]. That study concluded that CD34+ myeloid leukemia cells are characterized with elevated $\mathrm{HH}$ activity, as they express the downstream effector genes GLI1 and GLI2, and HH inhibition should be pursued as a therapeutic strategy in the treatment of AML [42].

\section{Wnt inhibition}

The Wnt signaling pathway includes a set of 19 highly conserved glycoproteins that serve as ligands for the 10 known frizzled (Fz) receptors. Two distinct Wnt pathways have been described: the canonical and noncanonical. The activation of the canonical pathway results in the accumulation of $\beta$-catenin in the cytoplasm, an effect that is not observed in the noncanonical pathway. $\beta$-Catenin, in turn, translocates to the nucleus, where it modulates the expression of Wnt target genes [36]. Aberrant Wnt activity has been observed in a variety of malignancies, including hepatocellular carcinoma, colon carcinoma, MM, AML, CML, and CLL [36].

Using CLL cells that display constitutive activation of the Wnt signaling pathway, salinomycin downregulated the expression of Wnt target genes such as LEF1 and cyclin D1. It decreased the survival of CLL cells, but had a minimal effect on human peripheral blood lymphocytes. In Wnt-transfected HEK-293 cells, salinomycin inhibited the phosphorylation of the Wnt coreceptor LRP6 and induced its degradation ( $\triangleright$ Figs. 2 and 3). The study concluded that drugs that inhibit the activity of Wnt signaling could impair the survival of cancer cells dependent on Wnt signaling [43].

Sulforaphane, an organosulfur compound with an isothiocyanate group, is naturally encountered in vegetables from the family Brassicaceae, such as broccoli or Brussels sprouts. Numerous studies have investigated the effects of sulforaphane on CSCs, and results demonstrated that it inhibited breast and pancreatic CSC growth by downregulating the Wnt pathway both in vitro and in vivo [44]. Importantly, sulforaphane targeted only the CSC population but no other tumoral cells [45]. In imatinib-resistant LSCs, sulforaphane reduced the expression of $\beta$-catenin and $A B C B 1$, induced apoptosis, and sensitized LSCs to the effects of imatinib ( $\vee$ Figs. 2 and 3). These studies suggested a potential role in the control of LSCs for sulforaphane, a molecule that was well tolerated in animal models [46].

\section{Notch inhibition}

Notch signaling is launched upon the binding of one of the five ligands (Jagged- 1 and -2, Delta-like-1, -3, and -4) to one of their cognate transmembrane receptors (Notch-1, $-2,-3$, and -4 ). Ligand binding induces Notch transmembrane receptor cleavage by $\gamma$ secretase and liberates the Notch intracellular domain, which then translocates to the nucleus and modulates the expression of Notch target genes $[47,48]$. Similar to Wnt and $\mathrm{HH}$, aberrant Notch activity has been reported in various malignant transformations, including metastasis, angiogenesis, and CSC maintenance. 
In a study conducted on clonotypic B cells derived from MM patients, Notch expression was reportedly high, suggesting a role of Notch in the maintenance of these MM-CSCs [49]. Treatment with a $y$-secretase inhibitor induced apoptosis of MM cells, enhanced the sensitivity to chemotherapy in vitro, and resulted in a significant antitumor effect in in vivo mouse xenografts and SCID-hu models of multiple myeloma, suggesting a novel role of Notch in the control of MM [50].

Curcumin, a bright yellow diarylheptanoid derivative isolated from Curcuma longa L. (Zingiberaceae), was demonstrated to play key roles in the control of cancer cell proliferation, migration, apoptosis, and metastasis. It also showed activity against pancreatic, breast, colon, and glioma cancer-derived CSCs [45]. Curcumin induced apoptosis and downregulated the expression of Notch target genes Hes-1 and Notch-1 in pancreatic cancer cells, suggesting that this natural compound modulates Notch signaling in pancreatic cancer ( $\triangleright$ Figs. 2 and 3) [51]. In addition, curcumin induced cell death in esophageal cancer cells and prostate cancer cells by inhibiting Notch [52, 53].

Although Notch inhibition has been demonstrated to be an effective strategy in the elimination of CSCs in certain LSC and MM-CSC models, Notch upregulation could play a tumor suppressive role in leukemia and in controlling the proliferation of MMCSCs $[54,55]$. Notch activation induced cell cycle arrest, differentiation, and death of acute myeloid LSCs both in vitro and in vivo [55]. In MM-CSCs, bruceantin, a quassinoid isolated from Brucea javanica, inhibited the proliferation of MM-CSCs in vitro ( $\triangleright$ Figs. 2 and 3). This effect was associated with an upregulation in Notch signaling [54].

\section{Telomerase inhibition}

Telomerase activity was demonstrated to be crucial for the maintenance of both NSCs and CSCs. Telomerases offer "immortality" and prevent cell senescence for human NSCs. Given that MM initiating cells share functional characteristics with NSCs, these CSCs exploit this characteristic for unlimited replicative potential. Telomerase activity was examined as a therapeutic target in MM, and it was found to regulate the clonogenic growth of MM-CSCs. A 72h treatment of human MM-CSCs isolated from cell lines and primary clinical specimens with imetelstat, a synthetic specific inhibitor of the reverse transcriptase activity of telomerase, inhibited clonogenic growth, induced MM-CSCs differentiation into plasma CD138 ${ }^{+}$cells, and reduced aldehyde dehydrogenase activity. A two-week treatment with imitelstat resulted in a significant reduction in telomere length and clonogenic MM growth both in vitro and in vivo, suggesting both long- and short-term telomerase inhibition effects on MM-CSCs [56]. Another study conducted in $\mathrm{AML}$ demonstrated that genetic deletion of the telomerase subunit Terc induced cell cycle arrest and apoptosis in LSCs. In addition, the pharmacologic inhibition of telomerase with imetelstat induced cell death in LSCs, and blocked leukemia progression and relapse following chemotherapy in mouse xenografts of human primary AML cells [57]. Taken together, the results of these studies demonstrate the potential of telomerase inhibition as a therapeutic strategy for the eradication of CSCs in both MM and leukemia.
In alignment with these studies, several natural products have been identified as telomerase inhibitors ( $\triangleright$ Figs. 2 and 3). Examples include compounds from microbial sources such as TLM, a macrocyclic alkaloid isolated from the bacteria Streptomyces anulatus, chrolactomycin, a compound found in Streptomyces sp. $569 \mathrm{~N}-3$ and rubromycins, a group of molecules with an uncommon 5,6-spiroketal core, extracted from various catinomycete species [58-60]. TLM has been demonstrated to be a very potent and selective telomerase inhibitor due to its remarkable selectivity for the G-quadruplex telomeric structures [61]. In one study, TLM impaired the maintenance and migration of patient-derived GSCs, and induced GSC apoptosis both in vitro and in vivo. Interestingly, the activity of TLM was minimal in normal neural precursors and non-GSCs, suggesting an important potential for TLM in the eradication of GSCs [62].

Telomerase inhibitors from plant sources are structurally diverse and include alkaloids such as the tetrahydroisoquinoline berberine, mainly isolated from different species of Berberidaceae or Papaveraceae, and cryptolepine, an indoloquinoline extracted from the roots of Cryptolepis sanguinolenta (Lindl.) Schltr. (Apocynaceae). Other inhibitors include the flavonoid quercetin, the sesquiterpene lactone helenalin (Arnica spp., Asteraceae), as well as oleic and gambogic acids [61]. The latter, a bright orange xanthonoid pigment, is extracted from the brownish resin of Garcinia hanburyi Hook. f. (Clusiaceae) and reduces telomerase activity by downregulation of the oncogene c-MYC, a transcription factor involved in the control of cell proliferation and differentiation [63].

The importance of telomerase activity in cancer and, particularly CSCs, offers a great opportunity to examine the potential of telomerase inhibition as a therapeutic strategy for the eradication of CSCs. Researchers have already identified plenty of telomerase inhibitors from natural sources, and ongoing research continues to identify inhibitors with novel and diverse chemical structures.

\section{Targeting Multiple Myeloma Cancer Stem Cells - Differentiation}

The differentiation potential of NSCs into several cell types poses the question: Could that key property of stem cells be exploited to control CSCs? The promotion of differentiation strips stem cells of their self-renewal, immortality, and detoxification tools, and sensitizes them towards standard therapies ( $\bullet$ Fig. 1). Currently, drug-induced differentiation is regarded as a promising approach to eliminate CSCs population. The proof of concept exists, and drug-induced terminal differentiation has been clinically used.

For instance, APL is characterized by the fusion protein PML/ RAR $\alpha$, which causes a differentiation blockade of PML-RAR $\alpha^{+}$cells and suppresses normal hematopoiesis. ATRA and arsenic trioxide were found capable of targeting PML/RAR $\alpha$ cells, and both compounds are capable of inducing complete remissions [64]. Interestingly, the mechanism by which ATRA induces remission is not cytotoxicity, but rather by inducing cellular differentiation ( $\triangleright$ Figs. 2 and 3). ATRA, a compound inspired from the natural product retinoic acid, reduces the proliferation of LSCs, and antagonizes the differentiation block caused by PML/RAR $\alpha$ fusion protein. This resulted in neutrophilic differentiation from APL 
blasts leading to the progressive replacement of malignant leukocytes by normal neutrophils, and therefore inducing complete remission within one year $[64,65]$.

The efficacy of ATRA-induced differentiation to target the stem cell-like tumor compartment in solid tumors remains elusive. ATRA has been shown to induce differentiation of GSCs, which resulted in therapy-sensitizing effects, and to disrupt GSCs motility in vitro. ATRA treatment alone also induced antitumor effects in vivo, demonstrating a potential for differentiation treatment to target the stem-like cell population in glioblastoma [66]. These results not only indicate that differentiation is possible for hematological malignancies, but it also warrants further investigation and serious efforts in MM.

In alignment with the results found with ATRA, a natural product that may induce the differentiation of MM-CSCs is withaferin A [67]. Withaferin A is a steroidal lactone isolated from Withania somnifera, which induced cell death in MM-CSCs. Specifically, withaferin A triggered cell cycle arrest, caused clear morphological changes and altered CSC-specific genes involved in lymphoid differentiation in MM-CSCs in vitro [67].

\section{Targeting Multiple Myeloma Cancer \\ Stem Cells - Migration}

Migration and invasion of MM cells within the BM remain only partly understood $[68,69]$. The contribution of MM-CSCs to this phenotype has not yet been addressed. However, if MM-CSCS share the same characteristics with CSCs derived from other tumors, then MM-CSCs would have a significant impact on the invasive phenotype observed within the BM of MM patients. Serious efforts need to be invested in the control of MM-CSCs invasiveness, and the identification of compounds capable of inhibiting this aspect of MM-CSCs may significantly affect the prognosis of MM patients. One natural product that has been implicated in the inhibition of LSCs invasion and migration is berberine. In addition to telomerase inhibition, berberine was shown to play a role in the control of cancer cell migration and invasion [70]. Berberine inhibited LSCs migration as well as the secretion of SDF-1, a homeostatic chemokine secreted by bone marrow stromal cells, suggesting a potential role for berberine in the control of LSCs migration ( $\triangleright$ Figs. 2 and 3) [70]. Another natural product that may be able to inhibit the migration of hematological CSCs is bruceantin. Using the classical in vitro scratch assay, bruceantin dose-dependently inhibited the migration of MM-CSCs [54].

\section{Conclusion}

Considering the relatively nontoxic profile of compounds such as curcumin and sulforaphane, the use of natural products represents a novel approach worth pursuing for targeting CSCs. The frequent observation that NPs sensitize CSCs to conventional therapies, combined with the fact that NPs can eliminate CSCs, further validates the notion that NPs possess diverse mechanisms of action that could be exploited to target numerous lethal characteristics of CSCs. However, further mechanistic and preclinical studies are required to eliminate potential toxicities associated with NPs, alone or when in combination therapy. Furthermore, due to similarities between NSCs and CSCs, the identification of compounds that selectively target CSCs remains a major challenge.

This review article aims to update the recent advancements achieved with regards to targeting MM-CSCs, particularly from its developmentally closest relative LSCS, and to draw lessons from other malignancies. In summary, it can be concluded that "targeting MM-CSCs with NPs" remains largely underexplored, and that NPs offer a tremendous reservoir of novel chemical scaffolds to be exploited.

\section{Conflict of Interest}

The authors declare no conflicts of interest.

\section{References}

[1] Agarwal JR, Matsui W. Multiple myeloma: a paradigm for translation of the cancer stem cell hypothesis. Anticancer Agents Med Chem 2010; 10: $116-120$

[2] San-Miguel JF, Hungria VT, Yoon SS, Beksac M, Dimopoulos MA, Elghandour A, Jedrzejczak WW, Gunther A, Nakorn TN, Siritanaratkul N, Corradini P, Chuncharunee S, Lee J], Schlossman RL, Shelekhova T, Yong K, Tan D, Numbenjapon T, Cavenagh JD, Hou J, LeBlanc R, Nahi H, Qiu L, Salwender H, Pulini S, Moreau P, Warzocha K, White D, Blade J, Chen W, de la Rubia J, Gimsing P, Lonial S, Kaufman JL, Ocio EM, Veskovski L, Sohn SK, Wang MC, Lee JH, Einsele H, Sopala M, Corrado C, Bengoudifa BR, Binlich F, Richardson PG. Panobinostat plus bortezomib and dexamethasone versus placebo plus bortezomib and dexamethasone in patients with relapsed or relapsed and refractory multiple myeloma: a multicentre, randomised, double-blind phase 3 trial. Lancet Oncol 2014; 15: 1195-1206

[3] Rajkumar SV. Treatment of multiple myeloma. Nat Rev Clin Oncol 2011; 8: 479-491

[4] Dalerba P, Cho RW, Clarke MF. Cancer stem cells: models and concepts. Annu Rev Med 2007; 58: 267-284

[5] Subramaniam D, Ramalingam S, Houchen CW, Anant S. Cancer stem cells: a novel paradigm for cancer prevention and treatment. Mini Rev Med Chem 2010; 10: 359-371

[6] Kyle RA, Rajkumar SV. Multiple myeloma. N Engl J Med 2004; 351: 1860-1873

[7] Drewinko B, Alexanian R, Boyer H, Barlogie B, Rubinow SI. The growth fraction of human myeloma cells. Blood 1981; 57: 333-338

[8] Huff CA, Matsui W. Multiple myeloma cancer stem cells. J Clin Oncol 2008; 26: 2895-2900

[9] Bergsagel DE, Valeriote FA. Growth characteristics of a mouse plasma cell tumor. Cancer Res 1968; 28: 2187-2196

[10] Hamburger A, Salmon SE. Primary bioassay of human myeloma stem cells. J Clin Invest 1977; 60: 846-854

[11] Basak GW, Carrier E. The search for multiple myeloma stem cells: the long and winding road. Biol Blood Marrow Transplant 2010; 16: 587-594

[12] Pilarski LM, Belch AR. Circulating monoclonal B cells expressing P glycoprotein may be a reservoir of multidrug-resistant disease in multiple myeloma. Blood 1994; 83: 724-736

[13] Pilarski LM, Hipperson G, Seeberger K, Pruski E, Coupland RW, Belch AR. Myeloma progenitors in the blood of patients with aggressive or minimal disease: engraftment and self-renewal of primary human myeloma in the bone marrow of NOD SCID mice. Blood 2000; 95: 1056-1065 
[14] Matsui W, Huff CA, Wang Q, Malehorn MT, Barber ], Tanhehco Y, Smith $\mathrm{BD}$, Civin $\mathrm{Cl}$, Jones RJ. Characterization of clonogenic multiple myeloma cells. Blood 2004; 103: 2332-2336

[15] Dean M, Fojo T, Bates S. Tumour stem cells and drug resistance. Nat Rev Cancer 2005; 5: 275-284

[16] Jakubikova J, Adamia S, Kost-Alimova M, Klippel S, Cervi D, Daley JF, Cholujova D, Kong SY, Leiba M, Blotta S, Ooi M, Delmore J, Laubach J, Richardson PG, Sedlak J, Anderson KC, Mitsiades CS. Lenalidomide targets clonogenic side population in multiple myeloma: pathophysiologic and clinical implications. Blood 2011; 117: 4409-4419

[17] Hawley TS, Riz I, Yang W, Wakabayashi Y, Depalma L, Chang YT, Peng W, Zhu J, Hawley RG. Identification of an ABCB1 (P-glycoprotein)-positive carfilzomib-resistant myeloma subpopulation by the pluripotent stem cell fluorescent dye CDy1. Am J Hematol 2013; 88: 265-272

[18] Lin MG, Liu LP, Li CY, Zhang M, Chen Y, Qin J, Gu YY, Li Z, Wu XL, Mo SL. Scutellaria extract decreases the proportion of side population cells in a myeloma cell line by down-regulating the expression of ABCG2 protein. Asian Pac J Cancer Prev 2013; 14: 7179-7186

[19] Gu YY, Liu LP, Qin J, Zhang M, Chen Y, Wang D, Li Z, Tang JZ, Mo SL. Baicalein decreases side population proportion via inhibition of ABCG2 in multiple myeloma cell line RPMI 8226 in vitro. Fitoterapia 2014; 94: 21-28

[20] Fuchs D, Daniel V, Sadeghi M, Opelz G, Naujokat C. Salinomycin overcomes $\mathrm{ABC}$ transporter-mediated multidrug and apoptosis resistance in human leukemia stem cell-like KG-1a cells. Biochem Biophys Res Commun 2010; 394: 1098-1104

[21] Januchowski R, Wojtowicz K, Zabel M. The role of aldehyde dehydrogenase $(A L D H)$ in cancer drug resistance. Biomed Pharmacother 2013; 67: 669-680

[22] Ginestier C, Hur MH, Charafe-Jauffret E, Monville F, Dutcher J, Brown M, Jacquemier J, Viens P, Kleer CG, Liu S. ALDH1 is a marker of normal and malignant human mammary stem cells and a predictor of poor clinical outcome. Cell Stem Cell 2007; 1: 555-567

[23] Croker AK, Allan AL. Inhibition of aldehyde dehydrogenase (ALDH) activity reduces chemotherapy and radiation resistance of stem-like ALDHhiCD44+ human breast cancer cells. Breast Cancer Res Treat 2012; 133: 75-87

[24] Tanei T, Morimoto K, Shimazu K, Kim S], Tanji Y, Taguchi T, Tamaki Y, Noguchi S. Association of breast cancer stem cells identified by aldehyde dehydrogenase 1 expression with resistance to sequential paclitaxel and epirubicin-based chemotherapy for breast cancers. Clin Cancer Res 2009; 15: 4234-4241

[25] Reghunathan R, Bi C, Liu SC, Loong KT, Chung TH, Huang G, Chng W]. Clonogenic multiple myeloma cells have shared stemness signature associated with patient survival. Oncotarget 2013; 4: 1230-1240

[26] Yang SM, Yasgar A, Miller B, Lal-Nag M, Brimacombe K, Hu X, Sun H, Wang A, Xu X, Nguyen K, Oppermann U, Ferrer M, Vasiliou V, Simeonov A, Jadhav A, Maloney DJ. Discovery of NCT-501, a potent and selective theophylline-based inhibitor of aldehyde dehydrogenase 1A1 (ALDH1A1). J Med Chem 2015; 58: 5967-5978

[27] Koppaka V, Thompson DC, Chen Y, Ellermann M, Nicolaou KC, Juvonen RO, Petersen D, Deitrich RA, Hurley TD, Vasiliou V. Aldehyde dehydrogenase inhibitors: a comprehensive review of the pharmacology, mechanism of action, substrate specificity, and clinical application. Pharmacol Rev 2012; 64: 520-539

[28] Lowe ED, Gao GY, Johnson LN, Keung WM. Structure of daidzin, a naturally occurring anti-alcohol-addiction agent, in complex with human mitochondrial aldehyde dehydrogenase. J Med Chem 2008; 51: 44824487

[29] Kikonyogo A, Abriola DP, Dryjanski M, Pietruszko R. Mechanism of inhibition of aldehyde dehydrogenase by citral, a retinoid antagonist. Eur J Biochem 1999; 262: 704-712

[30] Rekha GK, Sladek NE. Inhibition of human class 3 aldehyde dehydrogenase, and sensitization of tumor cells that express significant amounts of this enzyme to oxazaphosphorines, by the naturally occurring compound gossypol. Adv Exp Med Biol 1997; 414: 133-146

[31] Tottmar O, Lindberg P. Effects on rat liver acetaldehyde dehydrogenases in vitro and in vivo by coprine, the disulfiram-like constituent of Coprinus atramentarius. Acta Pharmacol Toxicol 1977; 40: 476-481

[32] Heydenreuter W, Kunold E, Sieber SA. Alkynol natural products target $\mathrm{ALDH} 2$ in cancer cells by irreversible binding to the active site. Chem Commun 2015; 51: 15784-15787

[33] Patlolla JM, Qian L, Biddick L, Zhang Y, Desai D, Amin S, Lightfoot S, Rao CV. $\beta$-Escin inhibits NNK-induced lung adenocarcinoma and ALDH1A1 and RhoA/Rock expression in $\mathrm{A} / \mathrm{J}$ mice and growth of $\mathrm{H} 460$ human lung cancer cells. Cancer Prev Res 2013; 6: 1140-1149

[34] Koppaka V, Thompson DC, Chen Y, Ellermann M, Nicolaou KC, Juvonen RO, Petersen D, Deitrich RA, Hurley TD, Vasiliou V. Aldehyde dehydrogenase inhibitors: a comprehensive review of the pharmacology, mechanism of action, substrate specificity, and clinical application. Pharmacol Rev 2012; 64: 520-539

[35] Michelot D. Poisoning by Coprinus atramentarius. Nat Toxins 1992; 1: $73-$ 80

[36] Takebe N, Harris PJ, Warren RQ, Ivy SP. Targeting cancer stem cells by inhibiting Wnt, Notch, and hedgehog pathways. Nat Rev Clin Oncol 2011; 8: 97-106

[37] O’Brien CA, Kreso A, Jamieson CH. Cancer stem cells and self-renewal. Clin Cancer Res 2010; 16: 3113-3120

[38] Duman-Scheel M, Weng L, Xin S, Du W. Hedgehog regulates cell growth and proliferation by inducing cyclin D and cyclin E. Nature 2002; 417 : 299-304

[39] Agren M, Kogerman P, Kleman MI, Wessling M, Toftgard R. Expression of the PTCH1 tumor suppressor gene is regulated by alternative promoters and a single functional Gli-binding site. Gene 2004; 330: 101-114

[40] Hui CC, Angers S. Gli proteins in development and disease. Annu Rev Cel Dev Biol 2011; 27: 513-537

[41] Peacock CD, Wang Q, Gesell GS, Corcoran-Schwartz IM, Jones E, Kim J, Devereux WL, Rhodes JT, Huff CA, Beachy PA, Watkins DN, Matsui W. Hedgehog signaling maintains a tumor stem cell compartment in multiple myeloma. Proc Natl Acad Sci U S A 2007; 104: 4048-4053

[42] Kobune M, Takimoto R, Murase K, lyama S, Sato T, Kikuchi S, Kawano Y, Miyanishi K, Sato Y, Niitsu Y, Kato J. Drug resistance is dramatically restored by hedgehog inhibitors in CD34+ leukemic cells. Cancer Sci 2009; 100: 948-955

[43] Lu D, Choi MY, Yu J, Castro JE, Kipps T], Carson DA. Salinomycin inhibits Wnt signaling and selectively induces apoptosis in chronic lymphocytic leukemia cells. Proc Natl Acad Sci U S A 2011; 108: 13253-13257

[44] Li Y, Zhang T, Korkaya H, Liu S, Lee HF, Newman B, Yu Y, Clouthier SG Schwartz SJ, Wicha MS. Sulforaphane, a dietary component of broccoli/ broccoli sprouts, inhibits breast cancer stem cells. Clin Cancer Res 2010; 16: $2580-2590$

[45] Wang Z, Ahmad A, Li Y, Azmi AS, Miele L, Sarkar FH. Targeting notch to eradicate pancreatic cancer stem cells for cancer therapy. Anticancer Res 2011; 31: 1105-1113

[46] Lin LC, Yeh CT, Kuo CC, Lee CM, Yen GC, Wang LS, Wu CH, Yang WC, Wu AT. Sulforaphane potentiates the efficacy of imatinib against chronic leukemia cancer stem cells through enhanced abrogation of Wnt/betacatenin function. J Agric Food Chem 2012; 60: 7031-7039

[47] Munoz P, Iliou MS, Esteller M. Epigenetic alterations involved in cancer stem cell reprogramming. Mol Oncol 2012; 6: 620-636

[48] Al-Hussaini H, Subramanyam D, Reedijk M, Sridhar SS. Notch signaling pathway as a therapeutic target in breast cancer. Mol Cancer Ther 2011; 10: 9-15

[49] Boucher K, Parquet N, Widen R, Shain K, Baz R, Alsina M, Koomen J, Anasetti C, Dalton W, Perez LE. Stemness of B-cell progenitors in multiple myeloma bone marrow. Clin Cancer Res 2012; 18: 6155-6168 
[50] Nefedova Y, Sullivan DM, Bolick SC, Dalton WS, Gabrilovich DI. Inhibition of notch signaling induces apoptosis of myeloma cells and enhances sensitivity to chemotherapy. Blood 2008; 111: 2220-2229

[51] Wang Z, Zhang Y, Banerjee S, Li Y, Sarkar FH. Notch-1 down-regulation by curcumin is associated with the inhibition of cell growth and the induction of apoptosis in pancreatic cancer cells. Cancer 2006; 106: 2503-2513

[52] Subramaniam D, Ponnurangam S, Ramamoorthy P, Standing D, Battafarano RJ, Anant S, Sharma P. Curcumin induces cell death in esophageal cancer cells through modulating Notch signaling. PLoS One 2012; 7: e30590

[53] Kong T, Wang Y, Xiao L, Liao L. Curcumin inhibits cell survival and migration by suppression of Notch-1 activity in prostate cancer cells. Int J Urol Nephrol 2013; 1: 35-39

[54] Issa ME, Berndt S, Carpentier G, Pezzuto JM, Cuendet M. Bruceantin inhibits multiple myeloma cancer stem cell proliferation. Cancer Biol Ther 2016; 17: 966-975

[55] Lobry C, Ntziachristos P, Ndiaye-Lobry D, Oh P, Cimmino L, Zhu N, Araldi E, Hu W, Freund J, Abdel-Wahab O, Ibrahim S, Skokos D, Armstrong SA, Levine RL, Park CY, Aifantis I. Notch pathway activation targets AML-initiating cell homeostasis and differentiation. J Exp Med 2013; 210: 301319

[56] Brennan SK, Wang Q, Tressler R, Harley C, Go N, Bassett E, Huff CA, Jones RJ, Matsui W. Telomerase inhibition targets clonogenic multiple myeloma cells through telomere length-dependent and independent mechanisms. PLoS One 2010; 5: e12487

[57] Bruedigam C, Bagger FO, Heidel FH, Kuhn CP, Guignes S, Song A, Austin $\mathrm{R}, \mathrm{Vu} \mathrm{T}$, Lee $\mathrm{E}$, Riyat $\mathrm{S}$. Telomerase inhibition effectively targets mouse and human AML stem cells and delays relapse following chemotherapy. Cell Stem Cell 2014; 15: 775-790

[58] Atkinson D], Brimble MA. Isolation, biological activity, biosynthesis and synthetic studies towards the rubromycin family of natural products. Nat Prod Rep 2015; 32: 811-840

[59] Nakai R, Kakita S, Asai A, Chiba S, Akinaga S, Mizukami T, Yamashita Y. Chrolactomycin, a novel antitumor antibiotic produced by Streptomyces sp. J Antibiot 2001; 54: 836-839

[60] Kim MY, Vankayalapati H, Shin-Ya K, Wierzba K, Hurley LH. Telomestatin, a potent telomerase inhibitor that interacts quite specifically with the human telomeric intramolecular G-quadruplex. J Am Chem Soc 2002; 124: 2098-2099

[61] Chen JLY, Sperry J, Ip NY, Brimble MA. Natural products targeting telomere maintenance. MedChemComm 2011; 2: 229-245

[62] Miyazaki T, Pan Y, Joshi K, Purohit D, Hu B, Demir H, Mazumder S, Okabe S, Yamori T, Viapiano M, Shin-ya K, Seimiya H, Nakano I. Telomestatin impairs glioma stem cell survival and growth through the disruption of telomeric G-quadruplex and inhibition of the proto-oncogene, c-Myb. Clin Cancer Res 2012; 18: 1268-1280

[63] Guo QL, Lin SS, You QD, Gu HY, Yu J, Zhao L, Qi Q, Liang F, Tan Z, Wang X. Inhibition of human telomerase reverse transcriptase gene expression by gambogic acid in human hepatoma SMMC-7721 cells. Life Sci 2006; 78: 1238-1245

[64] Puccetti E, Ruthardt M. Acute promyelocytic leukemia: PML/RARalpha and the leukemic stem cell. Leukemia 2004; 18: 1169-1175

[65] Werner B, Gallagher RE, Paietta EM, Litzow MR, Tallman MS, Wiernik PH, Slack JL, Willman CL, Sun Z, Traulsen A, Dingli D. Dynamics of leukemia stem-like cell extinction in acute promyelocytic leukemia. Cancer Res 2014; 74: 5386-5396

[66] Campos B, Wan F, Farhadi M, Ernst A, Zeppernick F, Tagscherer KE, Ahmadi R, Lohr J, Dictus C, Gdynia G, Combs SE, Goidts V, Helmke BM, Eckstein V, Roth W, Beckhove P, Lichter P, Unterberg A, Radlwimmer B, Herold-Mende C. Differentiation therapy exerts antitumor effects on stem-like glioma cells. Clin Cancer Res 2010; 16: 2715-2728

[67] Issa ME, Cuendet M. Withaferin A induces cell death and differentiation in multiple myeloma cancer stem cells. MedChemComm 2017; 8: 112121

[68] Hecht M, Heider U, Kaiser M, Von Metzler I, Sterz ], Sezer O. Osteoblasts promote migration and invasion of myeloma cells through upregulation of matrix metalloproteinases, urokinase plasminogen activator, hepatocyte growth factor and activation of p38 MAPK. Br J Haematol 2007; 138: $446-458$

[69] Qiang YW, Walsh K, Yao L, Kedei N, Blumberg PM, Rubin JS, Shaughnessy J, Rudikoff S. Whts induce migration and invasion of myeloma plasma cells. Blood 2005; 106: 1786-1793

[70] Li H, Guo L, Jie S, Liu W, Zhu J, Du W, Fan L, Wang X, Fu B, Huang S Berberine inhibits SDF-1-induced AML cells and leukemic stem cells migration via regulation of SDF-1 level in bone marrow stromal cells. Biomed Pharmacother 2008; 62: 573-578 